\title{
Improving the Inter-Laboratory Harmonization of the International Normalized Ratio (INR): Utilizing the Concept of Transference to Estimate and/or Validate International Sensitivity Index (ISI) and Mean Normal Prothrombin Time (MNPT) Values and/or to Eliminate Measurement Bias
}

\author{
EMMANUEL J FAVALORO, WENDY MCVICKER, YIFANG ZHANG, SAYED HAMDAM, \\ MICHELLE HUYNH, PADMINI PERIS, MATTHEW O'NEAL, NAOMI HOCKER
}

BACKGROUND: The Prothrombin Time (PT) assay is clinically the most often requested coagulation test, as used primarily for monitoring of Vitamin $\mathrm{K}$ antagonist therapy where results are typically expressed as an International Normalized Ratio (INR). The INR reflects the patient's PT adjusted for the specific test reagent and instrument combination used by applying two correction factors, namely the International Sensitivity Index (ISI) and the Mean Normal Prothrombin Time (MNPT), according to the formula:

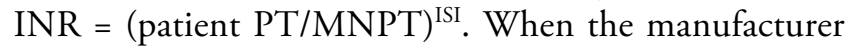
provides an ISI, laboratories are encumbered to check or locally validate the assigned value. Where a manufacturer does not provide an ISI, the laboratory needs to define its own (local ISI) value. The MNPT typically has to be locally defined, based on the population being tested. The main current CLSI recommendation for defining ISI values comprises use of commercial reference ('certified') plasma calibration sets, but FDA cleared material is limited, and different results may arise using different products. The MNPT can be defined using a WHO/CLSI recommended procedure requiring 20 normal individuals or with some calibration sets. Overall, there is limited data to validate the performance of these processes in laboratory practice, and ongoing evidence from external quality assurance (proficiency testing) programs indicates continued failure in INR harmonization, suggesting that ISI and MNPT values used by laboratories (and presumably assessed using current recommended processes) continue to be inaccurate.

OBJECTIVE: To assess some novel approaches to the laboratory estimation and/or validation of ISI and
MNPT values for use in the INR calculation, and including the process of 'transference', normally used to assess the comparability of analytical systems or to transfer reference intervals between comparable systems.

RESULTS: We have successfully adapted these comparative procedures, including 'transference', to permit ongoing estimation and/or validation of ISI and MNPT values for use in INR calculations for a range of instrumentation, which has led to improved harmonization of INR values obtained in our pathology network. These processes do not require the use of any normal individual plasmas or calibrator sets and greatly simplifies the INR process. Evidence for validation of the processes used is provided by ongoing satisfactory performance in external quality assurance (proficiency testing).

ABBREVIATIONS USED: Clinical and Laboratory Standard Institute (CLSI); external quality assurance (EQA); Prothrombin Time (PT); International Normalized Ratio (INR); International Sensitivity Index (ISI); Mean Normal Prothrombin Time (MNPT); Vitamin K antagonist(s) (VKA(s)); Vitamin $\mathrm{K}$ antagonist therapy (VKAT).

INDEX TERMS: Prothrombin Time; International Normalized Ratio; International Sensitivity Index; Mean Normal Prothrombin Time; harmonization; standardization.

Clin Lab Sci 2012;25(1):13 
Emmanuel J Favaloro BSc (Hons), PhD, FFSc (RCPA), Institute of Clinical Pathology and Medical Research (ICPMR), Westmead Hospital, Westmead

Wendy McVicker BAppSc (Med Lab Sc), Institute of Clinical Pathology and Medical Research (ICPMR), Westmead Hospital, Westmead

Yifang Zhang Dip Health Sc (Pathology), Institute of Clinical Pathology and Medical Research (ICPMR), Westmead Hospital, Westmead

Sayed Hamdam BSc, Haemotology, Pathology, West, Blacktown Hospital, Blacktown

Michelle Huynh BSc, Institute of Clinical Pathology and Medical Research (ICPMR), Westmead Hospital, Westmead

Padmini Peris BSc, Grad Dip in Clin Chem, MSc (Clin Chem), Institute of Clinical Pathology and Medical Research (ICPMR), Westmead Hospital, Westmead

Matthew O'Neal BMedSc, Nepean \& Blue Mountains Pathology Service, Lithgow District Hospital, Lithgow

Naomi Hocker BAppSc (Med Lab Sc), Haematology Department, South West Pathology Service, Wagga Wagga

Address for Correspondence: E.J. Favaloro, BSc (Hons), PhD, FFSc (RCPA), Department of Haematology, Institute of Clinical Pathology and Medical Research (ICPMR), Westmead Hospital, WESTMEAD, NSW, 2145, AUSTRALIA. (612) 9845 6618, Fax: (612) 9689 2331, emmanuel.favaloro@swahs.health.nsw.gov.au

\section{INTRODUCTION}

The Prothrombin Time (PT) assay is clinically the most often requested coagulation test, as used primarily for monitoring of Vitamin $\mathrm{K}$ antagonist therapy (VKAT). For example, over 30 million outpatient prescriptions for warfarin were reportedly written in the United States in 2004 according to a national prescription audit. ${ }^{1}$ The PT for any given individual on VKAT will vary widely according to the reagent and instrument used to perform the test, and accordingly the International Normalized Ratio (INR) system was devised to harmonize test results and to adjust for the variation otherwise arising. ${ }^{1}$ The INR reflects the patient's PT adjusted for the specific test reagent and instrument combination used by the laboratory by applying two correction factors, namely the 'International Sensitivity Index' (ISI) and the 'Mean Normal Prothrombin Time' (MNPT), and according to

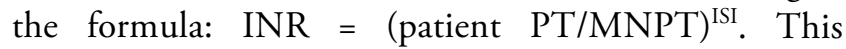
process substantially reduces the inter-laboratory variation otherwise obtained using the $\mathrm{PT}$, as for example evidenced by external quality assurance (EQA; or proficiency testing) data for samples tested using a wide variation of reagents and instrument combinations, and thus improves the clinical management of patients undergoing VKAT. ${ }^{1}$ Nevertheless, variation in INR values still exists, as again evidenced by recent EQA data, ${ }^{2-4}$ suggesting ongoing problems with INR standardization or harmonization.

There are only three components to an INR. Accordingly, any variation in INR values generated on the same plasma sample tested by different laboratories must derive from one or a combination of these components. The variation in any patient's PT attributable to a specific reagent and instrument combination can easily be estimated and is essentially expressed by the assay's imprecision, typically by means of intra- and inter- assay variation. Notably, given modern instrumentation and a laboratory's use of appropriate internal quality control processes, assay imprecision for PT values, reflecting analytical events, are well known to be quite low (generally $<5 \%$ as a coefficient of variation).

In contrast, ISI and MNPT values may be considered as 'extra-analytical variables'. 1,5 While some manufacturers provide assigned ISI values for specific PT reagents and instrumentation, primarily their own material, the vast number of possible reagent/instrument combinations would preclude this in most situations. Even should the manufacturer provide a generic or instrument specific ISI value, laboratories are still encumbered to check (or locally validate) the assigned value. ${ }^{6}$ Where a manufacturer does not provide an ISI, the laboratory needs to define its own (local ISI) value. ${ }^{6}$ Given the complexity of the WHO recommended procedure for defining or validating ISI values, the main current CLSI recommendation comprises the use of commercial reference-plasma calibration sets (called 'certified plasmas' in CLSI language). ${ }^{6}$ However, this is made difficult in the USA since FDA cleared material is currently limited to a single manufacturer for use on a 
single platform. ${ }^{7}$ In any case, evidence suggests that different ISI estimates may arise when a laboratory uses different commercial products, ${ }^{8}$ leading to uncertainty regarding which ISI value is most correct or should be adopted by the laboratory.

The MNPT typically has to be locally defined, based on the population being tested. The MNPT can be defined using a WHO and CLSI recommended procedure requiring 20 normal individuals ${ }^{6}$ or with some calibration (certified plasma) sets. $^{8}$ Again, evidence indicates that different MNPT values will be generated using different commercial products and with different sets of 20 normal individuals, ${ }^{8}$ again generating uncertainty regarding which MNPT value is most correct or should be used by the laboratory.

Overall, then, the variation in INR values currently observed from EQA or proficiency testing programs, reflecting cross-laboratory testing of identical plasmas, would indicate some continued failure in INR harmonization, and suggest that ISI and MNPT values assigned by laboratories, as presumably estimated or validated using current (e.g., CLSI) recommended processes, may continue to be inaccurate. We have thus initiated additional and alternate processes in our institution, as also recently assessed in other sites within our pathology network, and based on various comparative assessments, and including the conceptual process of transference, to estimate and/or validate local laboratory ISI and MNPT values, and we report our most recent experience here.

\section{MATERIALS AND METHODS}

\section{Setting}

Our Pathology network is detailed in Figure 1. The Institute of Clinical Pathology and Medical Research (ICPMR) is the main campus of our pathology network (called 'Pathology West'). The ICPMR pathology campus provides the general pathology needs of Westmead Hospital, a 975 bed tertiary level academic teaching hospital, as well as specialized pathology services to all network laboratories within Pathology West (currently 28 in total) and to a wide range of other referral centers. Local network laboratories provide local general pathology needs to local hospitals, general practitioners, and other local clients. By landmass, Pathology West provides the largest coverage of any other pathology provider in the state of New South Wales, Australia; this currently exceeds 75\% coverage of the state's geography. The ICPMR campus also provides scientific and technical support for all network laboratories. The current report provides an assessment of the effectiveness of the described processes, including transference, in relation to ISI, MNPT and INR harmonization, and using recent representative findings from the main ICPMR campus and a few network laboratories used as case studies.

\section{Overview of methods available for establishing and/or verifying values for ISI and MNPT}

There are many methods available for establishing and/or verifying values for ISI and MNPT for use in the INR test system. For the purpose of this report, these have been differentially identified as CLSI recommended procedures ${ }^{6}$ and our novel 'investigative' procedures, with the latter representing the research thrust of the current report.

CLSI recommended procedures: As outlined in the relevant CLSI document, ${ }^{6}$ the MNPT is defined as the geometric mean of the PTs of the healthy adult population and can be approximated by the geometric mean of the PT calculated from at least 20 fresh samples from healthy individuals, including those of both sexes. The WHO recommended procedure for establishing an ISI requires the testing of 60 samples from individuals stabilized on VKAT plus 20 individual normal samples that need to undergo testing with the trial (or test) PT reagent versus an international reference thromboplastin. Although potentially used by manufacturers of PT reagents to generate 'generic' or reagent/instrument specific ISI values for related manufacturer PT reagents and instrumentation, this is an onerous and complex procedure that is not normally undertaken by hemostasis laboratories to establish local ISI values. CLSI considers local laboratory verification of a manufacturer assigned generic ISI value to be mandatory, and also strongly recommends local verification of instrument specific ISI values. ${ }^{6}$ For this purpose, CLSI recommends the use of certified plasmas, supplemented by testing performed through EQA (proficiency testing) programs. An example of the certified plasma approach is given in Figure 2 . Appreciably, there are many commercially available plasma sets now available to permit verification (or derivation) of a local ISI, with some of these also facilitating the verification (or derivation) of a MNPT value. ${ }^{8}$ However, to date, there is only a single FDA cleared system. ${ }^{7}$ 
Investigative procedures: Our laboratory has previously reported on some major problems associated with the above noted recommended approaches. ${ }^{1,2,8}$ Specifically: (i) although the use of 20 individual normal plasmas is considered acceptable in order to provide a local laboratory MNPT value, a significant variation in actual MNPT values can be obtained with different sets of 20 individual plasmas (range of 12.7 - 14.2 seconds from the original previously published investigation for our specific instrument and reagent combination); ${ }^{8}$ (ii) different commercial calibration plasma sets (or certified plasmas in CLSI language) will also give rise to widely different ISI and MNPT values (range of 1.12 to 1.30 for ISI and 13.8 to 14.4 for MNPT for one specific reagent and instrument combination as previously reported). ${ }^{8}$ Accordingly, we have previously investigated and reported on the potential utility of alternate methods for local verification of ISI and MNPT values. The major investigative approach utilizes comparative regression analysis, utilizing an 'existing' versus 'replacement' thromboplastin reagent evaluation, to estimate both the ISI and MNPT of the replacement reagent as compared with the existing reagent. ${ }^{1,2,8}$ Conceptually, this process is analogous to the process of transference, normally used to define or transfer reference ranges after assessment of the comparability of test systems. ${ }^{9}$ The process as applied by our organization has consistently shown promise in regards to better harmonization of the INR using peer-laboratories and EQA data as comparison. ${ }^{1,2,8}$ Furthermore, a comparison of new reagents/batches with existing reagents/batches prior to their incorporated use in the laboratory is a normal or standard practice procedure. For PTs, local comparisons typically involve performing parallel testing of the existing reagent batch versus the new 'trial' ('replacement' or 'test') reagent batch using a large number (typically >100) of plasma samples covering a wide range of PT values. Further details are reported in the Results section to highlight recent findings.

An additional investigative method involves the use of recent EQA data, and specifically linear regression analysis using local PT values plotted against EQA median data used as the 'reference'. Again, further details are provided in results to highlight recent findings, but this procedure is primarily used to assess and eliminate bias in INR values across our pathology network. Notably, the elimination of bias is an important component of quality improvement according to the ISO 15189 standard the quality and competence of medical laboratories. ${ }^{10}$

\section{Local historical perspective}

The current report communicates on recent activities related to the above investigative procedures, as undertaken within our Pathology Network. Given that our laboratory has published previously in this area, ${ }^{1,2,8} \mathrm{a}$ brief synopsis of the local history may be of use to help differentially identify the updated findings from those previously reported. The ICPMR laboratory has been involved in INR testing since our facility opened in the early 1980s. Our facility also hosted the 'Australasian Reference Thromboplastin (ART) Unit' for many years in the late 1980s to mid-1990s. The ART Unit provided the main thromboplastin reagent in use in Australasian laboratories for PT and INR testing during that period, utilizing the WHO recommended procedure for establishing the ISI of its reagent. In latter years, the ART unit also generated ISI calibration sets (certified plasmas) for use by Australasian laboratories to verify local ISI and MNPT values. The ART Unit was at all times closely aligned to the Royal College of Pathologists of Australasia (RCPA) Haematology Quality Assurance Program (QAP), which provides Australasian pathology laboratories with EQA (proficiency testing) services.

The ART unit was also closely aligned to the ICPMR hemostasis laboratories, which both assisted in local validation testing of ART reagents, as well as utilizing these reagents for pathology testing. The original thromboplastin reagent manufactured by the ART Unit and thus utilized by our ICPMR laboratory and earlier network laboratories was human brain derived. This was later changed to a human placental product. After the ART Unit was disbanded, the ICPMR laboratory switched to a commercially available but similar reagent (i.e., replaced like with like), and namely the Thromborel-S reagent from Dade-Behring (as it was then known). PT testing was performed manually for VKAT patients at local clinics, and using a Coag-a-mate $\mathrm{XM}$ instrument (from BioMerieux at that time) for other samples. The ICPMR laboratory, and the local metropolitan network, has continued to use the Thromborel-S reagent (now from Siemens) since that time, although instrumentation has progressed from the Coag-a-mate XM to an ACL-300R (Instrumentation Laboratories) to an MDA-180 ${ }^{11}$ (then from AKZO) to current instrumentation comprising a STA-R Evolution 
(Stago). Of relevance to the current report are our most recent publications relating to the investigative procedures, ${ }^{1,2,8}$ and most recently pertaining to studies performed in 2008 and 2009. ${ }^{2}$ Notably, the Thromborel-S reagent is provided with generic and instrument specific ISI values, but the manufacturer does not provide an instrument specific ISI value for Stago instrumentation, including the STA-R Evolution. The current report primarily relates to the most recent studies undertaken between 2008 and 2011, and also includes some representative EQA data for these years.

\section{RESULTS}

Case Study 1: Verifying or Establishing a local ISI and MNPT at the ICPMR Main Campus Laboratory

As noted in methods, the major investigative procedure involves performing a regression analysis using the existing thromboplastin reagent (used as 'reference') versus the new (trial, test or replacement) reagent, with the data plotted using the log of the INR for the existing reference reagent on the $\mathrm{x}$-axis, and the log of the PTs using the new 'trial' reagent on the y-axis. The latest three such studies undertaken in the main campus ICPMR laboratory is shown in Figure 3, and explained in the figure legend. The approach can be used to locally verify an existing manufacturer provided ISI value, or in the absence of an instrument specific ISI value to establish a local ISI value, or to verify an ISI established using calibration sets (certified plasmas). The approach can also be used to verify a MNPT value established using the WHO/CLSI recommended procedure, or using calibration sets (certified plasmas), or else to establish a local MNPT value. Given our confidence in this procedure, as validated in previous studies, ${ }^{1,2,8}$ current evaluations to establish local ISI and MNPT values as then further verified using EQA data, has since 2007 been performed without any further verification or use of calibration (certified) plasma sets or normal individual plasmas. That the local ISI and MNPT values generated by this investigative procedure are valid is highlighted by ongoing satisfactory EQA (proficiency test) data, essentially showing ongoing equivalence to peer median values (Figures 4 and 5). As noted in Methods, this procedure has now been in use in this laboratory for over 5 years, and including five changes in reagent lots. However, it is important to note that this requires the initially existing methodology (i.e., existing PT reagent lot used as reference) to have a truly validated ISI and MNPT value.
Case Study 2. Establishing and Verifying local ISI and MNPT values at Metropolitan Network Laboratories

A similar approach is now used in many of our network laboratories to verify or establish a local ISI and MNPT, depending on the site in question, the reagent in use, and the local instrumentation. For example, those laboratories that use Thromborel-S reagent and Stago instrumentation (which currently comprise all the metropolitan laboratories), use a similar process to that of the main ICPMR campus, to estimate ISI and MNPT values which are then verified using EQA data. A sample set smaller than that used in the main laboratory is often employed in the smaller laboratories.

An example for a medium sized metropolitan laboratory using a Stago Compact instrument is shown in Figure 6. This laboratory services the needs of a 400-bed hospital, and obtained its first Stago Compact in late 2008. It was decided that local ISI and MNPT values could be derived for a planned new PT reagent lot by local testing on the Stago Compact as compared to testing of the same samples at the Westmead ICPMR campus using the Stago STA-R and the then existing (and ISI \& MNPT validated) PT reagent in use (Figure 6A). This assessment was facilitated by regular courier transport between, and the geographical proximity of, the two metropolitan laboratories. The locally derived ISI and MNPT values were verified by EQA prior to incorporated use, and thereafter a similar process to the main campus ICPMR laboratory has been employed to continue the process of establishing local ISI and MNPT values (example shown in Figure 6B), with ongoing satisfactory EQA performance (Figure 6C).

Other examples of the above process comprise smaller metropolitan laboratories using Thromborel $S$ reagent and the smaller START-4 analyzers. These similar approaches have yielded ongoing satisfactory EQA performance as also shown in Figure 6C.

Case Study 3. Verifying local ISI and MNPT values in a medium sized Network Country Laboratory.

In a recent publication, ${ }^{2}$ we gave an example of a country laboratory servicing a medium sized ( 256 bed) country hospital using a CA-540 instrument with Thromborel-S PT reagent. Given that both the instrument and reagent is provided by a single manufacturer (Siemens), a generic ISI is provided. However, when the laboratory attempted to locally verify the manufacturer provided generic ISI (0.96) 
using a certified plasma set from the same manufacturer, a different ISI value was obtained (1.07). A local regression analysis was therefore undertaken, which provided an ISI (1.02) closer to that of the generic ISI, and which also provided a MNPT estimate (12.1). Interestingly, a subsequent repeat evaluation using another lot of a certified plasma set from the same manufacturer generated very similar ISI (1.01) and MNPT values (12.3). Use of the laboratory local assigned values (representing the latter values) has provided ongoing evidence of satisfactory EQA performance (Figure 7). In this case example, then, the local regression analysis provided a means to verify the ISI and MNPT values obtained with the (second) certified plasma set.

Case Study 4. Eliminating bias in INR reporting from a small Network Country Laboratory.

Our last case study example involves a small country laboratory in which bias in INR reporting was raised as a possible issue for laboratory accreditation. This laboratory uses a Coag-a-mate XM analyser (BioMerieux) with Recombiplastin reagent (Instrumentation Laboratory), and used a commercial calibration (certified) plasma set to establish the local ISI (1.18) and MNPT (10.8). These values led to a slight bias in INR values compared to peer laboratories according to RCPA QAP median data (Figure 8A), and although this bias was not particularly large we recognized that this could be easily eliminated using procedures analogous to those described above, using comparative assessment of local vs RCPA data. Thus, the log of the local laboratory PT values were plotted (on the y-axis) against the log of the RCPA QAP median INR values (used as 'reference' or consensus on the $\mathrm{x}$-axis) (Figure 8B), to generate a regression line that permitted re-establishment of the ISI (1.21) and the MNPT (11.5). Continued use of the new ISI and MNPT values was later shown to have eliminated bias as predicted by our prior experience (Figure $8 \mathrm{C}$ ).

\section{DISCUSSION}

The INR system as used for VKAT monitoring remains problematic as evidenced by ongoing high variability in EQA (proficiency test) data, as previously published ${ }^{2-4}$ and as also shown in Figure 4 for our local geography. The most likely explanation is that laboratories continue to utilize inaccurate ISI and MNPT values for their INR calculations, and despite the availability of CLSI and WHO recommended procedures. ${ }^{6}$ In brief, the recommended procedures, although clearly shown by past study to improve standardization, ${ }^{12,13}$ are unfortunately not infallible, and in practice will not necessarily provide consistent findings from evaluation to evaluation. ${ }^{1,2,8}$ Moreover, although several new oral anticoagulants are emerging in clinical practice, threatening at least a reduction in VKAT, ${ }^{14,15}$ the INR test system is likely to remain in clinical use for the foreseeable future for a variety of reasons. Accordingly, laboratories will still need to grapple with the INR for at least the decade to come, and thus alternate and validated procedures for estimating and/or verifying ISI and MNPT values additional to current recommendations ${ }^{6}$ would be of value to all laboratories involved in INR testing.

Our laboratory has therefore evaluated and now adopted several novel approaches to facilitate this process, primarily employing regression analysis to permit derivation of both ISI and MNPT values, essentially by a process that is conceptually similar to that of transference. ${ }^{9}$ Indeed, our ongoing use of this process, and ongoing satisfactory performance in EQA at the main campus ICMPR laboratory (Figures 4 and 5), has seen us continue this process without any further use of commercial calibration plasma (certified plasma) sets (for ISI and MNPT validation) or normal individual plasmas (for MNPT validation). This process has been in place for over 5 years with over 5 changes in reagent lot. A similar or modified process is now in use in many of our network laboratories, which currently comprise 28 laboratories of differing size, instrumentation and reagent use. Although our longterm goal is to standardise methodologies and instrumentation, this requires a large amount of resourcing to replace instrumentation to that of a standard platform.

The approach we currently undertake is largely analogous to that of using certified calibration plasmas, excepting that there are a much larger number of data points. For the process to work, several postulates are required, including that the existing reagent and instrumentation combination, intended to be used as the 'reference' test system for the evaluation, has previously been 'validated' (i.e., is therefore suitable) for such purpose (including a correctly assigned ISI and MNPT). Therefore, the success of this process is incumbent on some prior validation, as previously highlighted. ${ }^{1,2,8}$ In our specific case, this validation has 
been achieved through a well devolved history of association with the ART Unit and RCPA Haematology QAP, and also by using a series of calibration plasma sets from various suppliers together with many normal individuals for estimation of an MNPT. ${ }^{1,2,8}$ Initial and ongoing validation has also been evidenced by ongoing acceptable performance in EQA (Figures 4 and 5). The process has also subsequently been successfully adapted to other laboratories in our pathology network, and is increasingly proving its worth in terms of harmonization of the INR across this network.

\section{CONCLUSION}

No single procedure for estimating an ISI and/or MNPT can be considered definitive. Accordingly, the process of estimation, calibration, validation and assignment of ISI and MNPT values should be considered a multi-step process, with a summary of a recommended approach shown as an algorithm in Figure 9. The processes described in this report have proven of considerable value to our local laboratory network in terms of harmonization of INR results, and will thus also be expected to improve harmonization of results within other laboratory networks. Nevertheless, given the investigational nature of such processes, it would be prudent to locally validate these before their broader application. For example, our experience with local regression analysis as a means to estimate/validate ISI and MNPT values is largely limited to one thromboplastin reagent, and the behaviour of all PT reagents may not be identical. Thus, like the case for the WHO based procedure, such a process may best be applied for the comparison of like to like reagents, or perhaps even to different lot batches of the same reagent. In any case, we look forward to supportive verification studies in the future.

\section{SUMMARY OF RECOMMENDATIONS}

1. If a manufacturer assigns an ISI for your laboratory's reagent and instrument combination, we recommend that the laboratory adopt that ISI as an interim estimate and then locally check (validate or verify) the correctness of this assigned ISI value prior to its formal adoption. This may simply comprise ongoing selfassessment and adequate performance in EQA (proficiency testing). A possible supplementary approach would be to undertake a local regression analysis of 'existing versus replacement' reagent and/or an evaluation using a commercial calibration (certified plasma) set (see Figure 9).

2. It is sometimes necessary or convenient for laboratories to use a thromboplastin that does not have an ISI specific for the instrument in use or even for the method of clot detection (e.g., optical versus mechanical). In this situation, the laboratory must either locally calibrate the PT reagent or else use an alternate method to determine the ISI. This can be achieved by evaluation of either a commercial calibration (certified plasma) set or a local regression analysis of 'existing versus replacement' reagent, and then an alternative validation procedure should be applied (i.e., as per Figure 9 and Recommendation 1 above).

3. MNPT values should be estimated as per CLSI and WHO guidelines, ${ }^{6}$ or using a local linear regression analysis as highlighted in this report, but with an additional validation step (Figure 9). From our experience, it is strongly suggested that 20 normal individuals may not provide a true estimate of MNPT, ${ }^{8}$ and on-going assessment of (and adequate performance in) comparative peer-group EQA or proficiency testing is essential.

4. Always use two independent steps for the initial 'estimation' and subsequent 'validation' procedure, and ensure that values generated from the different approaches match. If this is the case, the ISI and MNPT can be considered to be 'validated' and the laboratory can then adopt these values for local use. If the values do not match, then one is likely to be in error, and a second validation step is then required.

ACKNOWLEDGEMENTS: The authors would like to acknowledge and thank Bronwen Yensch, Kim Day and various members of staff from the main ICPMR campus CORE laboratory for gathering data and technical assistance respectively. 


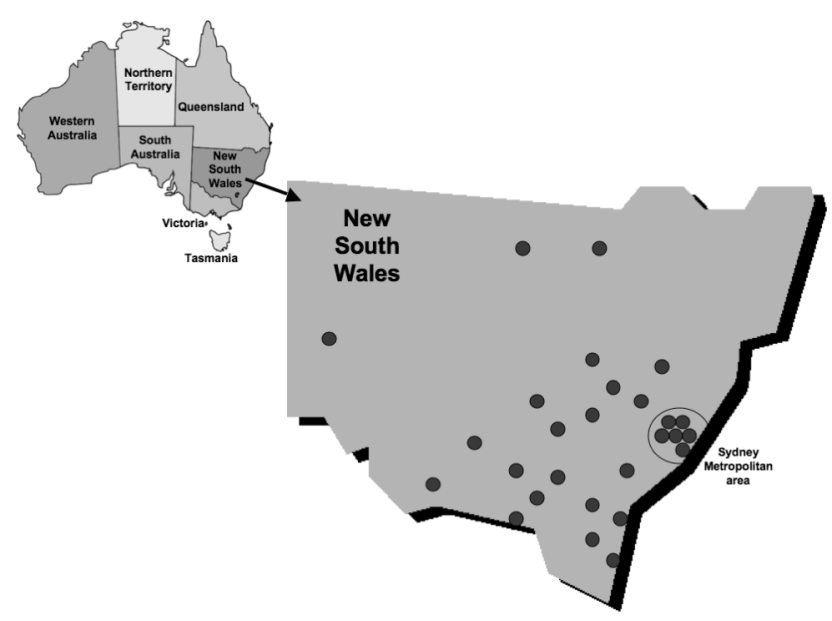

Figure 1: Study setting. Pathology West comprises 28 network laboratories and covers $>75 \%$ of the landmass of the state of New South Wales, within Australia. The ICPMR campus comprises the major metropolitan site for pathology within the network

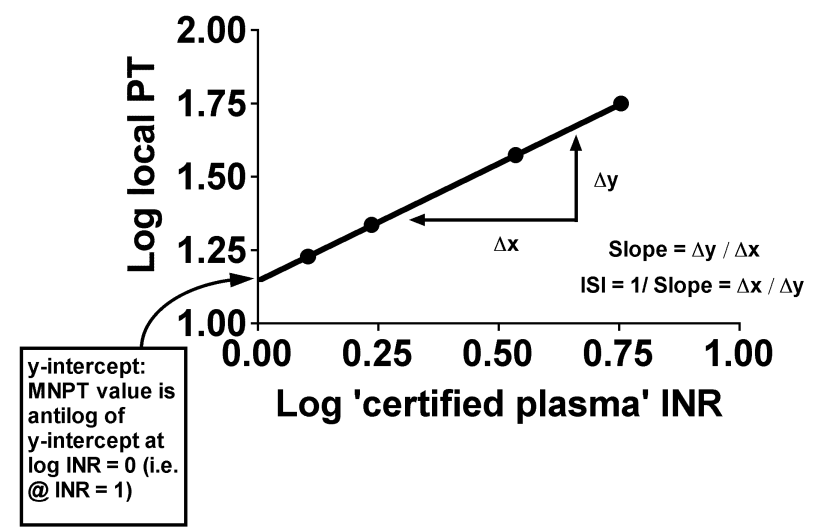

Figure 2: Calibration (certified) plasma set local verification of ISI and MNPT values. An outline of the process undertaken to verify or establish a local ISI and MNPT value using these plasma sets is as follows: The calibration (certified plasma) set provides a number of plasma samples each assigned a 'reference' INR value pertaining to a particular methodology. The laboratory locally tests these plasmas using the laboratory thromboplastin reagent, and then is able to plot the values obtained with the local reagent as $\log$ PTs on the $y$-axis against the log of the INR values assigned to the calibration set on the $\mathrm{x}$-axis. The local ISI is then derived from the slope (as 1/slope) of the line and the MNPT can be derived (with some commercial sets) from the $y$-intercept (viz; the local MNPT value is the antilog of the $y$-intercept at $\log I N R=0$ (i.e. at INR $=1$ )). In some cases, the laboratory provides the PT data to the manufacturer of the certified plasma set, who will in turn provide the laboratory the ISI (and sometimes a MNPT) value from the data provided; however, the above essentially outlines the process used.

\section{A. $2008-05$}

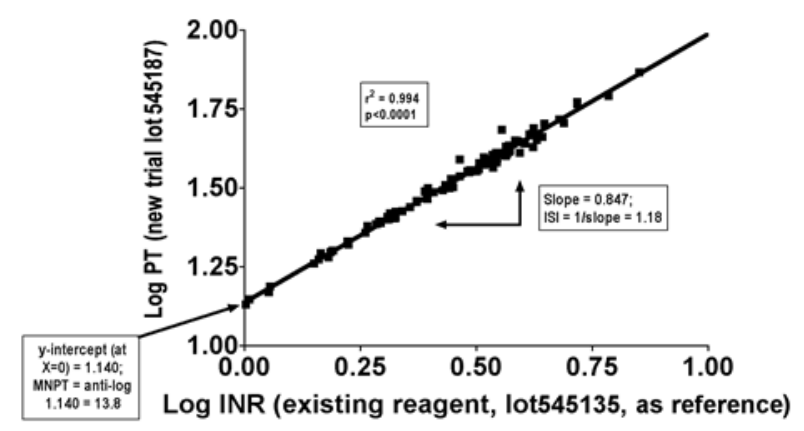

B. 2009-07

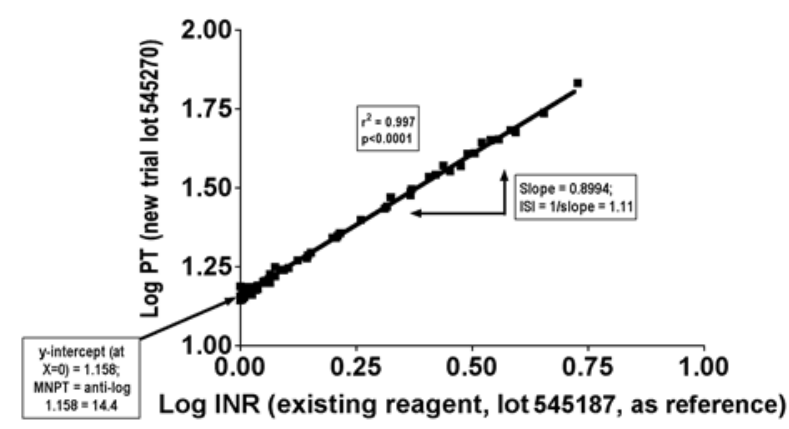

C. 2011-01

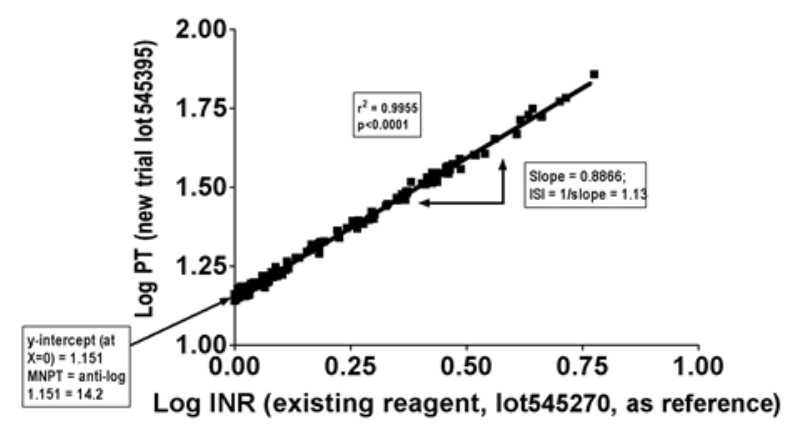

Figure 3. Verifying or establishing ISI and MNPT values from linear regression data ('transference' method; Case study 1). An outline of the process undertaken to verify or establish a local ISI and MNPT value using the investigative procedure highlighted in the current report is as follows: a large number of patient test samples are assessed for PT values using the existing thromboplastin reagent (used as a 'reference' reagent) as well as the new (planned replacement, test or trial) reagent. For our main laboratory, a minimum of 100 samples are run, with PT values reflecting a wide range from normal to high INR values. The laboratory is then able to plot the values obtained with the trial reagent as $\log$ PTs on the y-axis against the $\log$ of the INRs obtained using the existing reagent (used as 'reference') on the $\mathrm{x}$-axis. The local ISI is then derived from the slope (as 1/slope) of the line and the MNPT can be derived from the y-intercept (viz; as per 
the certified plasma model, the local MNPT value is the antilog of the $y$-intercept at $\log I N R=0$ (i.e. at INR = 1)). This figure represents data from the three most recent evaluations, being early-2008 (Figure A), mid-2009 (Figure B), and early 2011 (Figure C), and the main campus (ICPMR) laboratory Stago STA-R Evolution instrument using Thromborel-S reagent in each case. In Figure A, the new thromboplastin reagent at that evaluation (lot \#545187; expiry 01-2011) was compared against the existing thromboplastin reagent at that time (lot \#545135), to obtain a derived ISI and MNPT for the new reagent lot of 1.18 and 13.8 respectively (against those of the previous reagent lot, respectively 1.09 and 13.7). Similarly, in Figure B, the new thromboplastin reagent at that evaluation (lot \#545270; expiry 01-2011) is compared against the existing thromboplastin reagent at that time (lot \#545187; expiry 01-2011), to obtain a derived ISI and MNPT for the new reagent lot of 1.11 and 14.4 respectively (against those of the previous reagent lot, respectively 1.18 and 13.8). Lastly, in Figure $\mathrm{C}$, the new thromboplastin reagent (lot \#545395; expiry 03-2013) is compared against the existing thromboplastin reagent at that time (lot \#545270; expiry 01-2011), to obtain a derived ISI and MNPT for the new reagent lot of 1.13 and 14.2 respectively (against those of the previous reagent lot, respectively 1.11 and 14.4 ).

A. Accuracy Analysis

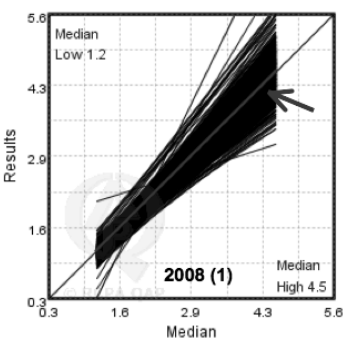

C. Accuracy Analysis

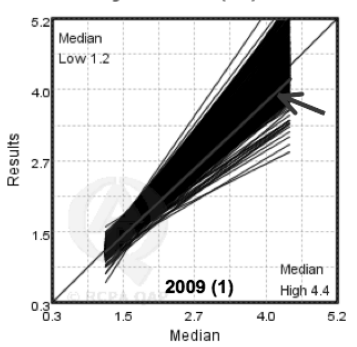

E. Regression Lines (695)

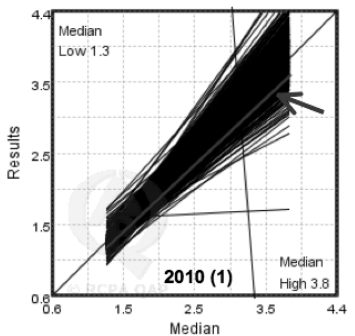

B. Accuracy Analysis

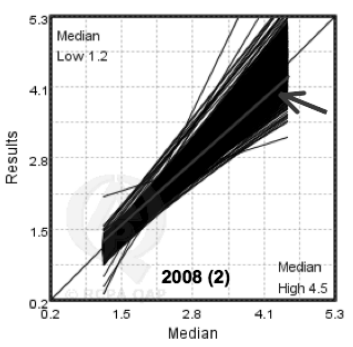

D. Accuracy Analysis

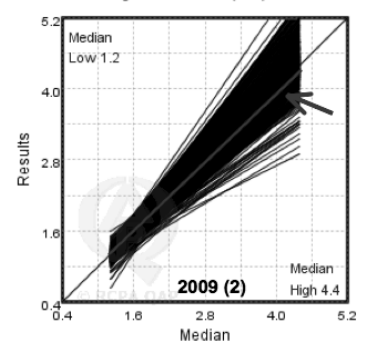

F. Accuracy Analysis

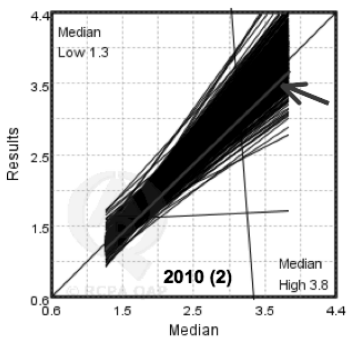

Figure 4. Verification of ISI and MNPT values established from linear regression ('transference') data using EQA (proficiency testing) data (part 1). Composite of EQA (proficiency test) data from 2008 to 2010 from RCPA Haematology QAP shown as 'end of year cycle' linear regression plots for INR for all participants. Data shown for complete years 2008 (Figures A and B), 2009 (Figures $\mathrm{C}$ and D) and 2010 (Figures E and F). Each figure plots the laboratory reported INR results (y-axis) vs the median reported INR (x-axis), with the ideal relationship reflected by the line of equivalence, which transects each figure from the lower left to upper right. In each year the RCPA QAP aims to provide samples that reflect a wide range of INR values; as shown in figures, this range covered $1.2-$ 4.5 (2008), 1.2-4.4 (2009) and 1.3-3.8 (2010). The results for each laboratory are plotted as individual regression lines $(\mathrm{n}=643$ in 2008, $\mathrm{n}=655$ in $2009, \mathrm{n}=695$ in 2010), and resultant data shows a wide spread with some laboratories showing significant positive or negative bias away from the ideal (line of equivalence). Occasional regression lines (see Figures E and F for 2010) do not follow expected patterns, potentially reflecting additional (e.g., transcription) errors in reporting. The local (main campus) ICPMR laboratory INR values plotted against $\mathrm{RCPA}$ reported median values are shown in each figure as a solid red line (arrowed). Data in each case shows near equivalence to median reported values, indicating good performance in this EQA (proficiency testing) setting. Figures $\mathrm{A}, \mathrm{C}$ and $\mathrm{E}$ reflect data for the main STA-R Evolution analyser, and figures $\mathrm{B}, \mathrm{D}$ and $\mathrm{F}$, the back-up STA-R Evolution analyser.

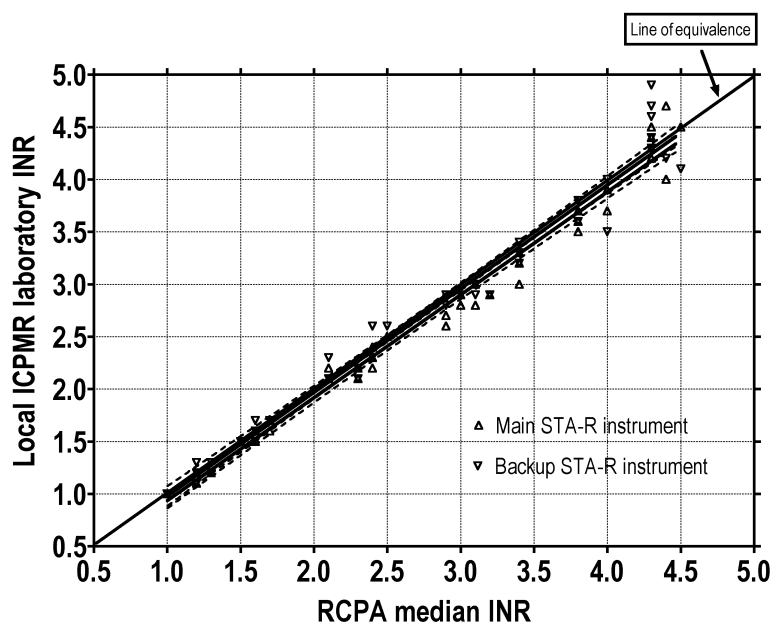

Figure 5. Verification of ISI and MNPT values established from linear regression ('transference') data using EQA (proficiency testing) data (part 2). Composite of EQA (proficiency testing) data from 2008 to current (July, 2011) showing local (main campus) ICPMR laboratory reported INR values plotted against median values from RCPA Haematology QAP. Data shown for both the main laboratory STA-R instrument, as well as the back up STA$\mathrm{R}$ instrument and include the $95 \%$ confidence intervals. As shown, data for both instruments overlaps the line of equivalence for the RCPA EQA data, and shows no 
significant bias. Individual data points away from the line of equivalence are reflective of assay variation (i.e., random error events), which is highest for high INR values.

\section{A. $2008-11$}

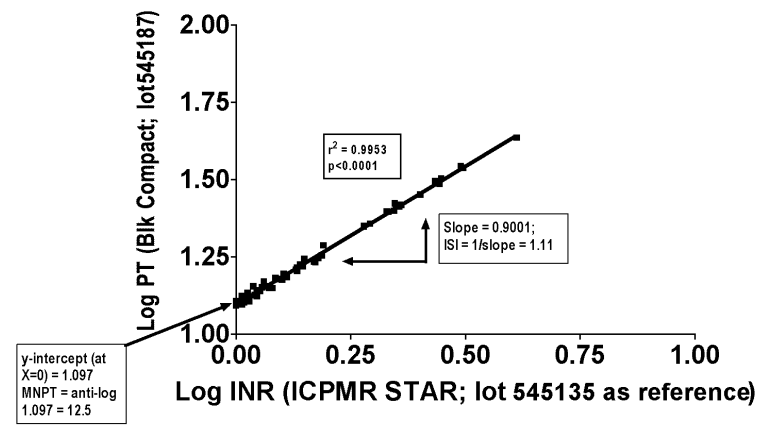

B. 2009-08
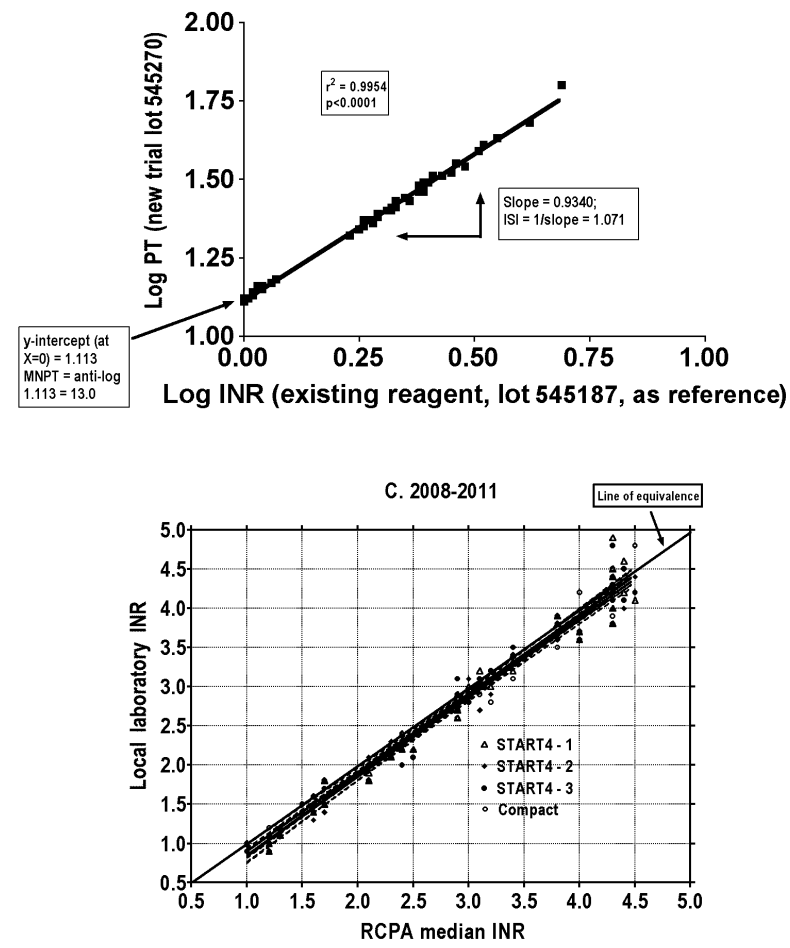

Figure 6. Verifying or establishing ISI and MNPT values from linear regression data ('transference' method; Case study 2). In this case, the laboratory is metropolitan based and geographically proximal to the main ICPMR campus. A new Stago Compact instrument was placed into the laboratory, and the first evaluation (Figure A; late 2008) assessed a large number of patient test samples for PT values using the existing thromboplastin reagent and instrument (Stago STA-R Evolution; used as the 'reference' test system) in place at the main ICPMR campus versus the new (test or trial) reagent and instrument (Stago Compact) at the second laboratory.
Like Case study 1 (Figure 3), a number of samples $(>50)$ are run, with PT values reflecting a wide range from normal to high INR values, and the values obtained with the trial reagent/instrument are plotted as log PTs on the $y$-axis against the $\log$ of the INRs obtained using the existing reagent/instrument (used as 'reference') on the $\mathrm{x}$ axis. The local ISI is then derived from the slope (as 1/slope) of the line and the MNPT can be derived from the y-intercept (viz; as per the certified plasma model, the local MNPT value is the antilog of the y-intercept at log INR $=0$ (i.e. at INR $=1)$ ). The subsequent evaluation (Figure B; mid-2009) was performed as per the normal procedure in use at the main campus (viz, as per Figure 3; using existing reagent vs replacement reagent on a single instrument, in this case a Stago Compact). Another evaluation has recently been conducted in early 2011 (data not shown). The verification for the ISI and MNPT values obtained by these procedures was obtained by adequate performance in EQA (proficiency testing), with data for 2008 to current identified in Figure C. Thus, reported INR data (with 95\% confidence interval) for the local laboratory plotted against the RCPA QAP median shows an overlap with the line of equivalence ('Compact'). This figure also shows EQA data for three other laboratory sites using Stago START-4 analyzers and the same regression analysis to define local ISI and MNPT values, with similar outcomes. Individual data points away from the line of equivalence are reflective of assay variation (i.e., random error events), which is highest for high INR values.

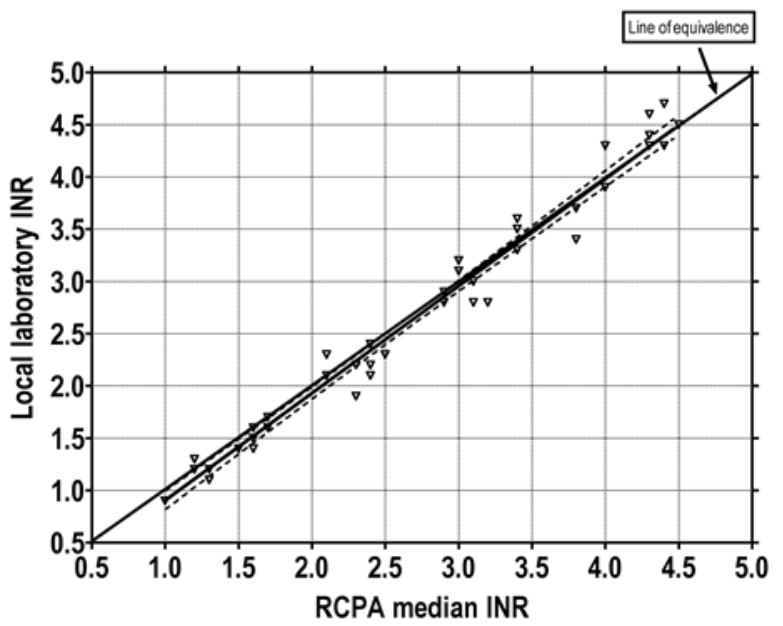

Figure 7. Verifying or establishing ISI and MNPT values using EQA (proficiency testing) data - Case study 3. As per previous figures, local laboratory reported INR values for a country based site have been plotted against the RCPA QAP median for the period 2008 to current (July, 2011) and data shows an overlap with the line of equivalence. Individual data points away from the line of equivalence are reflective of assay variation (i.e., random error events). 


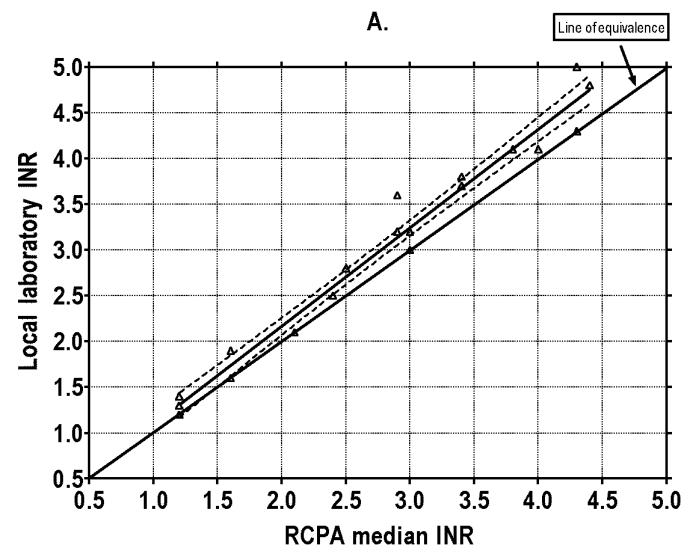

B.
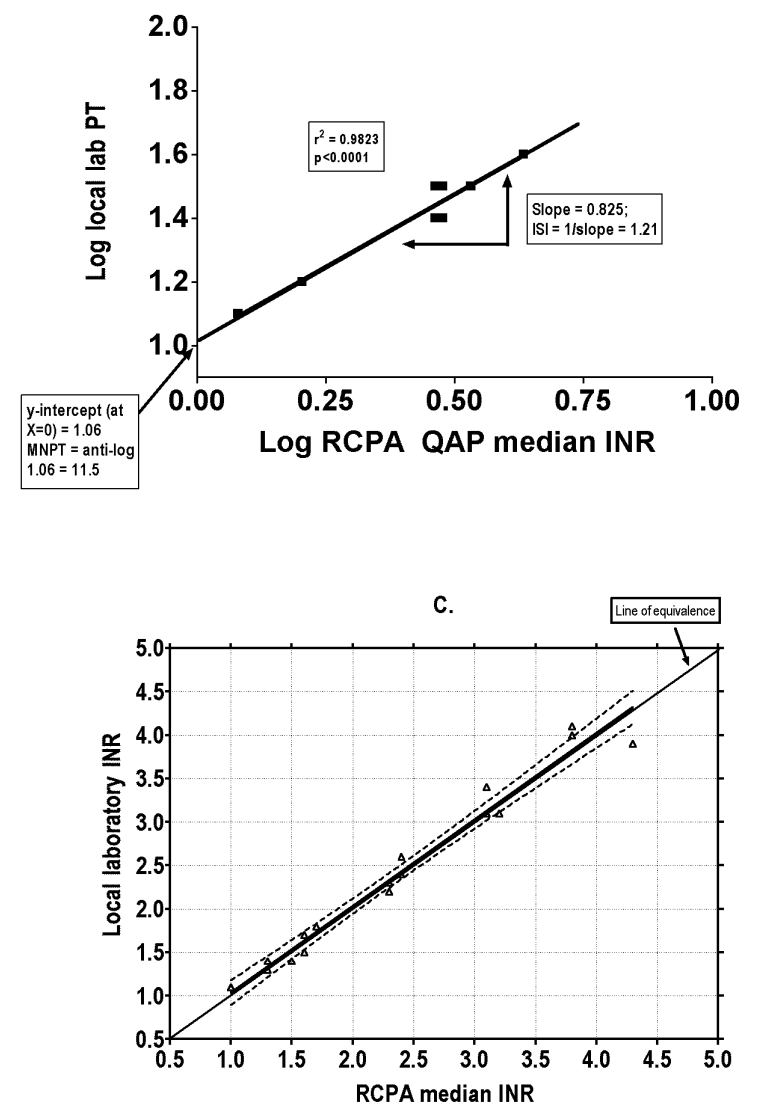

Figure 8. Eliminating bias in INR reporting from a small Network Country Laboratory - Case study 4 . In this case, local ISI and MNPT values were assigned for a Coag-a-mate XM analyser and Recombiplastin reagent using a commercial calibration (certified) plasma set; however, a positive bias was observed compared to peer laboratory performance in an EQA setting (Figure A). Revised ISI and MNPT values were obtained following a regression analysis, plotting the log of the local laboratory PT values against the log of the RCPA QAP median INR values (Figure B). Subsequent use of the revised ISI and MNPT values shows the elimination of bias and better harmonization with peer laboratory reported INRs (Figure C). 


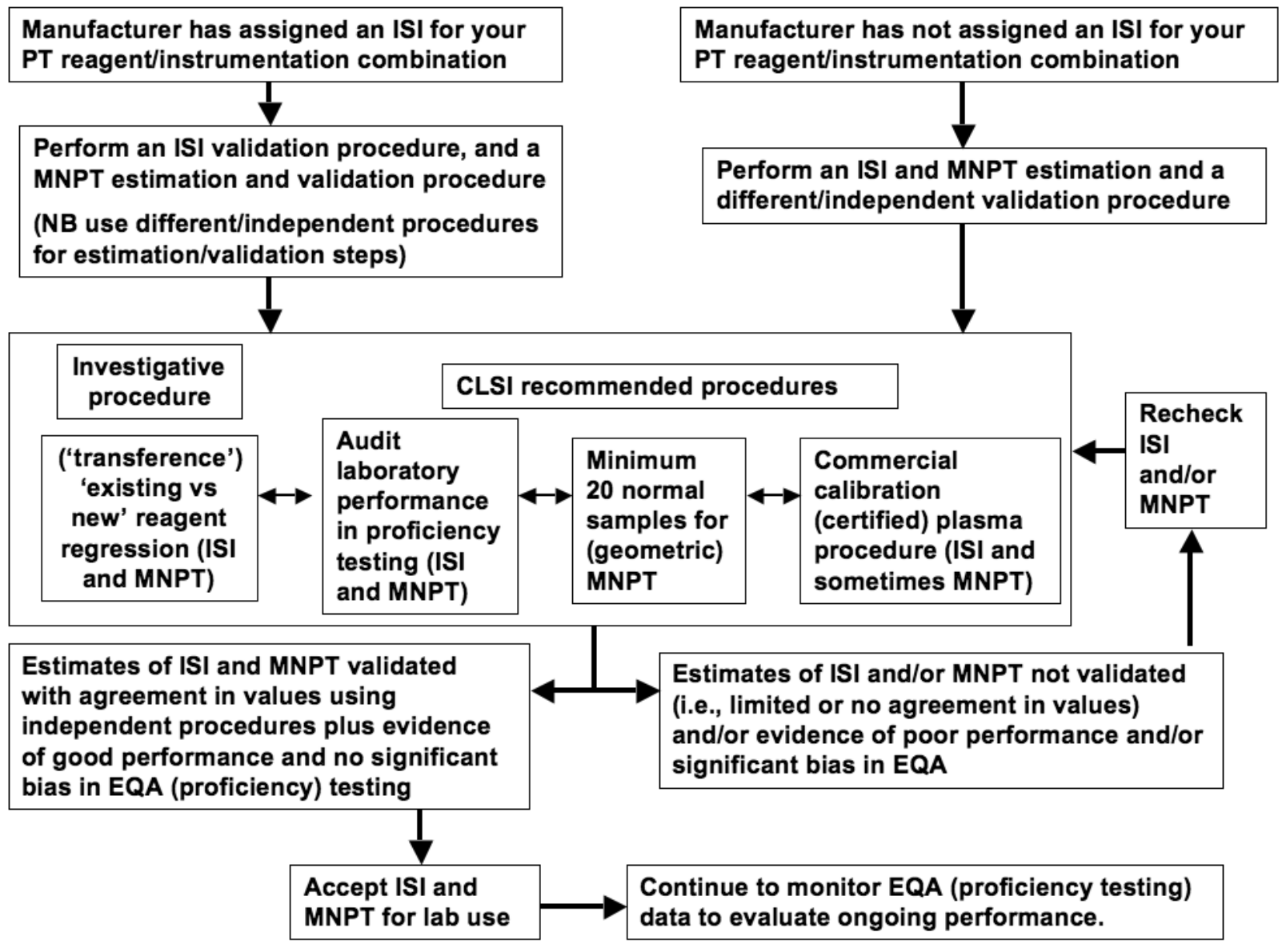

Figure 9: A recommended approach to the clinical laboratory calibration, estimation, validation and assignment of ISI and MNPT values for use in the INR system associated with VKAT. Adapted from Favaloro et al. ${ }^{1,2,8}$

\section{REFERENCES}

1. Favaloro EJ, Adcock DM. Standardization of the INR: how good is your laboratory's INR and can it be improved? Semin Thromb Hemost 2008;34:593-603.

2. Favaloro EJ, McVicker W, Hamdam S, Hocker N. Improving the harmonisation of the International Normalized Ratio (INR): Time to think outside the box? Clin Chem Lab Med, 2010; 48(8):1079-90.

3. Jennings I, Kitchen DP, Woods TA, Kitchen S, Walker ID. Emerging technologies and quality assurance: the United Kingdom National External Quality Assessment Scheme perspective. Semin Thromb Hemost 2007;33:243-9.

4. Cunningham MT, Brandt JT, Chandler WL, Eby CS, Hayes TE, Krishnan J, et al. Quality assurance in hemostasis: the perspective from the College of American Pathologists proficiency testing program. Semin Thromb Hemost 2007;33:250-8.

5. Favaloro EJ, Lippi G, Adcock DM. Preanalytical and postanalytical variables: the leading causes of diagnostic error in hemostasis? Semin Thromb Hemost 2008;34:612-34.

6. Adcock DM, Brien WF, Duff SL, Johnston M, Kitchen S, Marlar RA, et al. Procedures for validation of INR and local calibration of PT/INR systems; Approved Guideline. Nt H54A, Vol. 25 No. 23 (replaces H54-P Vol. 24 No. 30). Published by Clinical and Laboratory Standard Institute (CLSI; formally NCCLS). 2005, Wayne, Pennsylvania, USA.

7. HEMOSIL INR VALIDATE, HEMOSIL ISI CALIBRATE, ISIWEB (SOFTWARE); <http://www.accessdata.fda.gov/ scripts/cdrh/cfdocs/cfivd/index.cfm?db=pmn\&id=K090563> Accessed $19^{\text {th }}$ June 2011.

8. Favaloro EJ, Hamdam S, McDonald J, McVicker W, Ule V. Time to think outside the box? Prothrombin Time (PT), International Normalised Ratio (INR), International Sensitivity Index (ISI), Mean Normal Prothrombin Time (MNPT) and Measurement of Uncertainty (MU): A novel approach to standardisation. Pathology 2008;40:277-87.

9. Horowitz GL, Boyd JC, Ceriotti F, Garg U, Altaie S, Pesce A, et al. CLSI. Defining, Establishing, and Verifying Reference Intervals in the Clinical Laboratory; Proposed GuidelineThird Edition. CLSI document C28-P3. Wayne, PA: Clinical and Laboratory Standards Institute; 2008.)

10. ISO 15189. Medical laboratories - Particular requirements for quality and competence.

11. Mohammed A, Mehrabani PA, Coombs R, Molesworth L, and Favaloro EJ. The MDA-180 Coagulation Analyser: A laboratory evaluation. Pathology, 1997;29:176-83.

12. Adcock DM, Duff S. Enhanced standardization of the International Normalized Ratio through the use of plasma 


\section{RESEARCH AND REPORTS}

calibrants: a concise review. Blood Coag Fibrinolysis 2000;11:583-90.

13. McGlasson DL. A comparison of INRs after local calibration of thromboplastin international sensitivity indexes. Clin Lab Sci 2002;15(2):91-5.
14. Favaloro EJ, Lippi G. Editorial. Laboratory testing and/or monitoring of the new oral anticoagulants/antithrombotics: For and against? Clin Chem Lab Med. 2011;49(5):755-7.

15. Favaloro EJ, Lippi G, Koutts J. Laboratory testing of anticoagulants - the present and the future. Pathology, 2011;43:682-92.

The peer-reviewed Research and Reports Section seeks to publish reports of original research related to the clinical laboratory or one or more subspecialties, as well as information on important clinical laboratory-related topics such as technological, clinical, and experimental advances and innovations. Literature reviews are also included. Direct all inquiries to David $L$ McGlasson MS, MLS, 59th Clinical Research Division/SGRL, 2200Berquist Dr., Bldg. 4430, Lackland AFB TX 782369908,david.mcglasson@lackland.af.mil

Clinical Laboratory Science encourages readers to respond with thoughts, questions, or comments regarding these articles. Email responses towestminsterpublishers@comcast.net.In the subject line, please type the journal issue and lead author such as "CLIN LAB SCI 25(1) RE FAVALORO". Selected responses may appear in the Dialogue and Discussion section in a future issue. Responses may be edited for length and clarity. We look forward to hearing from you. 